\title{
Lympho-SPECT/CT as a tool to evaluate postoperative outcomes after LVA for lymphedema repair
}

\author{
Jose M. Lasso \\ Plastic and Reconstructive Surgery, Hospital General Universitario Gregorio Marañón, Madrid 28007, Spain. \\ Correspondence to: Dr. Jose M. Lasso, Plastic and Reconstructive Surgery, Hospital General Universitario Gregorio Marañón, C/ \\ Dr Esquerdo 46, Madrid 28007, Spain. E-mail: josemaria.lasso@salud.madrid.org
}

How to cite this article: Lasso JM. Lympho-SPECT/CT as a tool to evaluate postoperative outcomes after LVA for lymphedema repair. Plast Aesthet Res 2020;7:30. http://dx.doi.org/10.20517/2347-9264.2019.75

Received: 23 Dec 2019 First Decision: 19 Mar 2020 Revised: 29 Apr 2020 Accepted: 4 Jun 2020 Published: 18 Jun 2020

Science Editor: Xiao Long Copy Editor: Cai-Hong Wang Production Editor: Jing Yu

\begin{abstract}
Aim: To describe findings when comparing lympho-SPECT-CT images before and after lymphovenous anastomosis (LVA) surgeries and to correlate these results with pre- and post-operative volume changes in the limbs of patients.
\end{abstract}

Methods: An observational, prospective, longitudinal study was designed. 20 consecutive patients were treated for lymphedema by means of LVA between 2015 and 2018. All were affected by secondary lymphedema (ISG IIIII) following lymphadenectomy, radiation or both. All patients received preoperative rehabilitation as well as radiotherapy after oncological surgery. Limb volume was measured before surgery and at one year later. LVA was performed under general anesthesia with ICG guidance. ICG was also used to evaluate postoperative outcomes. Lympho-SPECT-CT was performed in all subjects at their first consultation and at one year after every surgical intervention. Description of findings included an absence of lymph nodes, new lymph node activity in anatomical areas and new lymphatic activity in extra-anatomical areas.

Results: Limb volume decreased in 19 patients after LVA. Six patients showed preoperative linear ICG patterns, combined with areas presenting with another type of pattern. After LVA, the linear pattern was observed in 11 patients. SPECT-CT/lymphoscintigraphy before surgery showed a total absence of lymph nodes, except in two cases, in whom small nodes in anatomical locations were described. After LVA, we observed new landmarks in 16 patients corresponding to lymphatic circulation that was not present in preoperative studies. In six cases, new

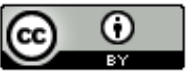

(C) The Author(s) 2020. Open Access This article is licensed under a Creative Commons Attribution 4.0 International License (https://creativecommons.org/licenses/by/4.0/), which permits unrestricted use, sharing, adaptation, distribution and reproduction in any medium or format, for any purpose, even commercially, as long as you give appropriate credit to the original author(s) and the source, provide a link to the Creative Commons license, and indicate if changes were made.

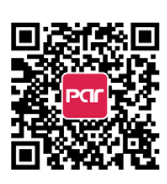


lymphatic activity compatible with lymph nodes was detectable after LVA. The Spearman correlation coefficient was negative when circumferences and lympho-SPECT-CT were tested $(P=0.02)$.

Conclusion: Results showed a postoperative decrease in volume that correlated inversely with lympho-SPECT/CT findings. Lympho-SPECT/CT provided additional information related to accurate identification and the anatomical location of lymphatic structures that were not observed before reconstructive surgery. It can be a complementary test to conventional lymphoscintigraphy.

Keywords: Lymphedema, supermicrosurgery, lymphovenous anastomosis, LVA, lympho-SPECT/CT, ICG

\section{INTRODUCTION}

Lymphedema is a debilitating disease in which drainage of the lymphatic system is impaired and affects a considerable number in the population. In developed countries, this is prevalent after pelvic or axillary radiotherapy, and lymph node dissection following oncologic surgery of the breast or pelvis. Unfortunately, secondary lymphedema and especially, primary lymphedema are still pathological conditions that cannot be treated definitively. Treatment prospects however, have improved for patients in the last decade due to new surgical techniques.

Current surgical treatments are based on microsurgical techniques ${ }^{[1-4]}$. The two main procedures are: lymph node transfer and lymphovenous anastomosis (LVA). Preoperative clinical evaluation of the patients and imaging studies of the lymphatic system are essential when surgery is planned for. On the other hand, postoperative imaging methods are crucial for understanding the efficacy of surgery.

Among these methods, lympho-SPECT/CT seems to be effective in evaluating lymphatic flow and to document lymph node regeneration. Previous publications have found that it should be a principal tool to complete functional evaluation of lymphedema ${ }^{[5]}$.

We report a prospective study in 20 patients with secondary lymphedema that was treated with LVA. The degree of lymphedema ranged from II to III (ISL classification). Our main goal was to describe new findings using lympho-SPECT-CT images before and after surgery and to correlate these results with the respective volume changes.

\section{METHODS}

An observational, prospective, longitudinal study was designed. 20 consecutive patients treated with LVA for lymphedema at the Hospital Gregorio Marañón in Madrid between 2015 and 2018 were included. All were affected by secondary lymphedema of the lower or upper limbs following surgical lymphadenectomy, radiation or both. Patients were randomly selected in out-patient hospital. The inclusion criteria were: secondary lymphedema of a limb; no previous reconstructive surgery of the lymphatic system; patients with previous rehabilitation therapy were considered for inclusion in the study; those with an ISL classification of lymphedema of degree II and II; and lymphedema had to be established for at least one year before reconstructive microsurgery.

\section{Clinical evaluation}

Pre- and postoperative evaluation was based on imaging and clinical examination. Limb circumference was measured at every $5 \mathrm{~cm}$ : point 1 - mid-palm or mid-foot, point 2 - bony landmarks (ulnar styloid process/lateral malleolus), C3-C 8 - every $5 \mathrm{~cm}$ above point 2 . The variable used for evaluation and followup was the circumference difference, which is equal to the sum of the differences between the involved 
and uninvolved limb at all points. The circumference difference was registered at first visit and at each postoperative visit after LVA. Results were considered stable and registered for analysis at the one-yearpostoperative visit after surgery.

The clinical examination also included a record of complications, episodes of cellulitis and an enquiry about subjective symptoms. Subjective perception of the disease was evaluated with a patient survey that consisted of four questions utilizing a five-point Likert-type scale ${ }^{[6]}$ and a fifth enquiry about overall treatment satisfaction (Satisfaction with limb weight, appearance, firmness, ability to perform daily activities: scores ranging from 1-5, being 5 the highest score; and would you undergo surgery again? yes/ no). The questionnaire was developed at our center and has been standardized for use in all lymphedema patients since 2008 .

\section{Surgical protocol (LVA-ICG)}

In the operating room, ICG (VERDYE ${ }^{\mathrm{TM}}$; Diagnostic Green GmbH, Aschheim-Dornach, Germany) was injected intradermally into the first and fourth web spaces $(0.1 \mathrm{~mL}$ per injection of a $25 \mathrm{mg} / \mathrm{mL}$ solution mixed with $5 \mathrm{~mL}$ of water for injection), 15 minutes later, circumferential fluorescent images of the lymphatic drainage channels were obtained using an infrared camera system (Photodynamic Eye, $\mathrm{PDE}^{\mathrm{TM}}$; Hamamatsu Photonics K.K., Hamamatsu, Japan). Lymphography patterns were described at this moment. Linear patterns or patterns that showed dynamic drainage of ICG were marked on the skin. ICG fluorescent images were obtained both before and during ICG-SL surgery and at every year postoperatively. Findings were labeled according to Yamamoto's dermal backflow classification and contrasted with postoperative SPECT-CT/lymphoscintigraphy results. It was found that this ICG lymphangiography staging system had a significant correlation with the clinical stage of lymphedema ${ }^{[7]}$. In the proximal part of the affected limb, dermal backflow patterns were more frequently found than linear patterns. The dermal backflow pattern is further divided into splash, stardust and diffuse patterns.

LVAs were performed under general anesthesia in all cases, with specific material and 12/0 monofilament sutures, under microscope magnification. Anastomoses were performed end-to-end and end-to-side, depending on the availability of recipient veins. Skin closure was achieved with superficial non-absorbable sutures $(4 / 0)$ without subcutaneous sutures.

\section{Postoperative care}

Oral antibiotics were prescribed for five days after surgery. Patients wore a soft non-elastic bandage that was replaced every 3 days for 3 weeks, under surgeons' supervision. Subsequently, from the fourth week, customized compression sleeves were placed for three months. Lymphatic drainage was performed twice a week during this period. After drainage, patients wore elastic stockings as instructed. On the other hand, conservative therapy prior to LVA consisted of lymphatic drainage that started three weeks before (twice per week).

\section{Nuclear medicine}

Lympho-SPECT/CT was performed in all subjects at the first consultation and at every year after surgical intervention. Lymphography was done with $46 \mathrm{MBq}$ Tc-99m of nanocolloidal human albumin (Nanocoll, GE Healthcare ${ }^{c}$ ) injected into the subcutaneous plane at the second web space in each limb (both healthy and affected) after dilution in $0.15 \mathrm{~mL}$ of $0.9 \%$ sodium chloride. As $85 \%$ of colloids are smaller than $80 \mathrm{~nm}$, a 30 -gauge needle was required. The interval between injections was less than $30 \mathrm{~s}$.

As patients may have severe alteration of lymphatic flow due to fibrosis or ectasia, they were asked to walk around or elevate the arms for 30 min before obtaining planar images. Conventional lymphoscintigraphy was then performed: anterior and posterior planar images from shoulder to hand (hip to foot) were 
acquired to obtain early (30 min) and late $(2 \mathrm{~h})$ images with a gamma camera $\left(\mathrm{GAMMG}{ }^{\mathrm{c}}\right)$ and low energyhigh resolution collimator (LEHR). The acquisition from each time point proceeded over $10 \mathrm{~min}$.

SPECT-CT images were acquired over $30 \mathrm{~min}$, starting at $3 \mathrm{~h}$ after injection of the nucleotide using a dual-head combined SPECT/CT camera (Optima ${ }^{\mathrm{TM}}$ NM/CT640, General Electric) with the following parameters: $128 \times 128$ matrix, rotation of $360^{\circ}, 4^{\circ}$ view angle, $25 \mathrm{~s}$ per projection; slice thickness was $2.5 \mathrm{~mm}$. Two nuclear medicine specialists reviewed all images regarding the detectability and number of lymph nodes.

Description of imaging findings included the absence of nodes, improved drainage of the lymph node basin, new lymphatic drainage in extra-anatomical position, and traces of the nucleotide constituting spots along the lymphatic system of the limb. Data acquired were compared with postoperative SPECT-CT/ lymphoscintigraphy results.

\section{Statistical analysis}

Categorical variables were described as numbers and percentages. Continuous variables were described as median (interquartile range). Lympho-SPECT-CT findings were described as: absence of nodes (0); traces of the nucleotide constituting spots along the lymphatic system of the limb (1); improved drainage of the lymph node basin (2); and new lymphatic drainage in extra-anatomical position - elbow, supraclavicular, parascapular (3). Correlation between continuous variables was evaluated using the Spearman coefficient. Statistical significance was defined as $P<0.05$. IBM SPSS software was used for calculation of the above.

\section{RESULTS}

The patients' age ranged from 25 to 76 years old, with a mean of 48.3 years. Sixteen and four patients had upper and lower limb lymphedema respectively. Eighteen patients were female.

The duration of disease from diagnosis to LVA surgery ranged from 15 months to 11 years. Fifteen patients had lymphadenectomy and postoperative radiotherapy (14 in the axilla and 1 in the groin); four had sentinel node biopsy and postoperative radiotherapy ( 2 axilla, 2 groin) and a single patient had radiotherapy (in the groin after pelvic surgery). A mean of 4.35 (range, 2-10) anastomoses were performed in each affected limb. In all cases, follow-up was more than 12 months after surgery (range, 12-24 months). All patients underwent preoperative rehabilitation, with different criteria. Six of them did not improve with non-surgical therapy while the rest worsened when therapy was discontinued [Table 1].

After LVA, all patients reported a subjective decrease in weight and/or firmness of the limb, and an improvement in their quality of life, with a mean increase in overall satisfaction of 4.3 points in a 20-point survey. The improvement in "satisfaction with limb appearance" was the most significant. All patients responded "yes" to the question "Would you undergo surgery again?" after completing sequential therapy (LVA and rehabilitation).

No severe postoperative complications have been reported to date. Four patients had frequent episodes of cellulitis preoperatively that required hospital admission (three per year or more). None of them have had repeated limb infection except an obese woman with lower limb lymphedema who smoked and did not follow postoperative recommendations.

With respect to volume decrease, the circumference measurements reached near-normal values (less than $6 \mathrm{~cm}$ difference) in 15 patients [Table 2]. 
Table 1. Progress is measured in months. When expressed in years, it is indicated along the table

\begin{tabular}{|c|c|c|c|c|c|c|c|}
\hline Patient & Age & Sex & Involved limb & ISL classification & Progress of lymphedema & Number of LVA & Preoperative rehabilitation \\
\hline 1 & 32 & $\mathrm{~F}$ & Upper right & II & 25 months & 3 & Temporarily effective \\
\hline 2 & 45 & $\mathrm{~F}$ & Upper right & II & 24 months & 3 & Temporarily effective \\
\hline 3 & 35 & M & Lower left & II & 3 years & 4 & Temporarily effective \\
\hline 4 & 41 & $\mathrm{~F}$ & Upper right & II & 18 months & 5 & Non effective \\
\hline 5 & 38 & $\mathrm{~F}$ & Upper left & II & 4 years & 4 & Temporarily effective \\
\hline 6 & 25 & $\mathrm{~F}$ & Lower right & III & 15 months & 4 & Non effective \\
\hline 7 & 56 & $\mathrm{~F}$ & Upper right & II & 25 months & 2 & Temporarily effective \\
\hline 8 & 39 & $\mathrm{~F}$ & Upper right & II & 11 years & 5 & Temporarily effective \\
\hline 9 & 45 & $\mathrm{~F}$ & Upper left & II & 3 years & 4 & Temporarily effective \\
\hline 10 & 67 & $\mathrm{~F}$ & Upper left & III & 40 months & 5 & Non effective \\
\hline 11 & 45 & $\mathrm{~F}$ & Lower right & II & 38 months & 6 & Temporarily effective \\
\hline 12 & 29 & M & Upper left & II & 3 years & 3 & Temporarily effective \\
\hline 13 & 76 & $F$ & Lower right & II & 28 months & 10 & Temporarily effective \\
\hline 14 & 50 & $\mathrm{~F}$ & Upper left & II & 24 months & 4 & Temporarily effective \\
\hline 15 & 62 & $\mathrm{~F}$ & Upper right & II & 4 years & 4 & Non effective \\
\hline 16 & 65 & $\mathrm{~F}$ & Upper left & III & 5 years & 5 & Non effective \\
\hline 17 & 60 & $\mathrm{~F}$ & Upper left & II & 6 years & 4 & Temporarily effective \\
\hline 18 & 49 & $\mathrm{~F}$ & Upper left & II & 25 months & 5 & Temporarily effective \\
\hline 19 & 53 & $\mathrm{~F}$ & Upper right & II & 23 months & 3 & Non effective \\
\hline 20 & 55 & $\mathrm{~F}$ & Upper right & II & 30 months & 4 & Temporarily effective \\
\hline
\end{tabular}

Temporarily effective: Patients worsened when non-surgical therapy was discontinued; Non effective: Patients non responders to nonsurgical therapy. LVA: lymphovenous anastomosis

Table 2. Results for each patient 1 year after LVA procedure (cm)

\begin{tabular}{lcccc}
\hline Patient & $\begin{array}{c}\text { Circumference difference } \\
\text { preLVA (cm) }\end{array}$ & $\begin{array}{c}\text { Circumference difference } \\
\text { postLVA (cm) }\end{array}$ & Satisfaction before surgeries & Satisfaction after surgeries \\
\hline 1 & 70 & 58 & 9 & 11 \\
2 & 7.3 & 6 & 10 & 12 \\
3 & 17 & 13.5 & 13 & 17 \\
4 & 11 & 6 & 12 \\
5 & 11.2 & 15 & 7 & 10 \\
6 & 17 & 22 & 9 & 15 \\
7 & 30 & 8.5 & 8 & 14 \\
8 & 10 & 39 & 6 & 10 \\
9 & 41 & 30 & 8 & 12 \\
10 & 37 & 22.5 & 7 & 16 \\
11 & 23 & 8 & 14 & 19 \\
12 & 10 & 12.5 & 11 & 17 \\
13 & 15 & 12 & 9 & 15 \\
14 & 18 & 41 & 11 & 17 \\
15 & 50 & 15 & 8 & 14 \\
16 & 20 & 43 & 14 & 16 \\
17 & 42 & 12.3 & 17 & 20 \\
18 & 15 & 21.2 & 16 & 19 \\
19 & 25 & 19 & 8 & 12 \\
20 & 22 & 30 & 12 & 18 \\
\hline
\end{tabular}

Satisfaction: sum of responses in survey before and after global treatment with lymphovenous anastomosis (LVA)

Figures 1 and 2 show volume reduction in the upper limb of a patient with lymphedema after breast cancer (patient 2).

Table 3 shows pre- and post-operative ICG and SPECT-CT/lymphoscintigraphy results for all patients in our study. Findings of SPECT-CT/lymphoscintigraphy before surgery showed a total absence of lymph 


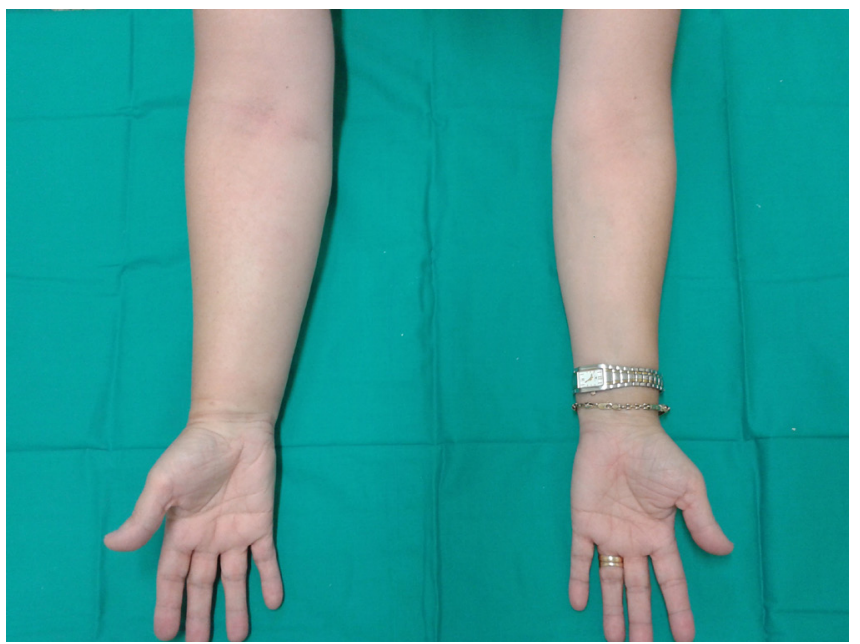

Figure 1. Pre-operative view of a ISL second degree lymphedema of the right upper limb showing a more affected cubital area. Red arrow

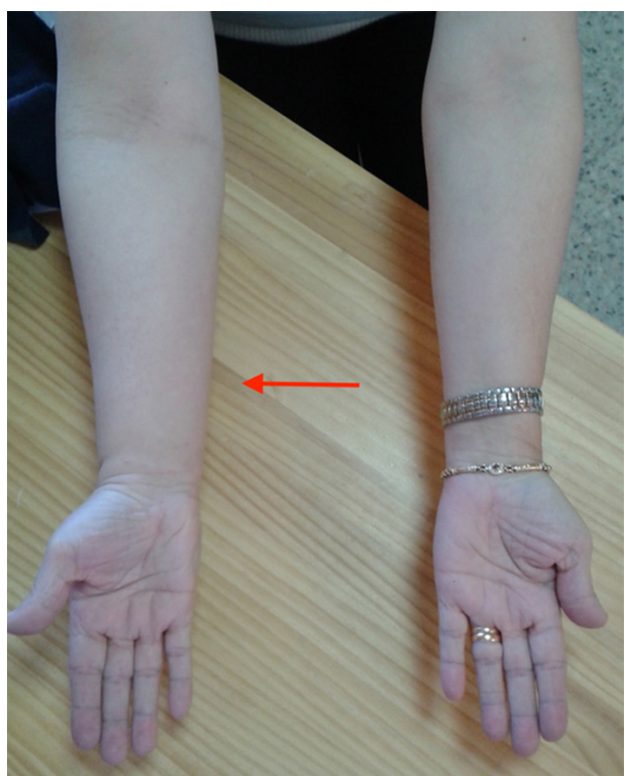

Figure 2. Post-operative view of a ISL second degree lymphedema of the right upper limb. The cubital area improved after lymphovenous anastomosis (red arrow).

nodes, except in two cases, in which several nodes in anatomical (axilla or groin) locations were described. These presented with a smaller size than the healthy, contralateral ones (Figure 3, patient 3).

In 16 patients, there were new landmarks corresponding to lymphatic circulation that were not present in pre-operative studies. In two patients, there were new spots that presented in unexpected anatomical areas, which may be considered as new lymphatic pathways. In six cases, images compatible with new functional lymph nodes were detectable after LVA (Figure 4, patient 14).

When ICG evaluation was performed before surgery, most patients presented with a diffuse or splash pattern. In six cases, there were segments of a linear pattern in combination with another pattern. One year later, several changes were observed in these patients. Eleven presented with a linear pattern, with four in combination with another pattern [Table 3]. Lymph nodes were not detected with this technique however. 
Table 3. Fluorescence patterns are registered according to the images precepted $15 \mathrm{~min}$ after ICG injection

\begin{tabular}{|c|c|c|c|c|}
\hline & PRE-LVA fluorescence pattern & $\begin{array}{c}\text { POST-LVA fluorescence } \\
\text { pattern }\end{array}$ & $\begin{array}{l}\text { Baseline SPECT-CT/ } \\
\text { lymphoscintigraphy }\end{array}$ & $\begin{array}{l}\text { Post LVA SPECT-CT/ } \\
\text { lymphoscintigraphy }\end{array}$ \\
\hline 1 & Diffuse/stardust & Stardust & - & - \\
\hline 2 & Diffuse/stardust & Diffuse/linear in some areas & - & ++ \\
\hline 3 & Diffuse/stardust & Stardust & - & + \\
\hline 4 & Stardust & Linear & - & $+^{\star}$ \\
\hline 5 & Stardust & Linear & - & + \\
\hline 6 & Linear, stardust & Linear - slow progression & - & + \\
\hline 7 & Linear/stardust & Linear & - & ++ \\
\hline 8 & Diffuse & Diffuse & - & + \\
\hline 9 & Stardust/splash & Splash & - & + \\
\hline 10 & Linear/splash & Linear & + & + \\
\hline 11 & Linear/stardust & Linear & - & ++ \\
\hline 12 & Diffuse & Diffuse & - & + \\
\hline 13 & Splash & Splash & - & - \\
\hline 14 & Stardust & Linear/splash & - & $++^{\star}$ \\
\hline 15 & Diffuse/splash & Splash & - & + \\
\hline 16 & Diffuse & Linear/diffuse & - & - \\
\hline 17 & Linear/stardust & Linear & - & ++ \\
\hline 18 & Stardust & Stardust & - & + \\
\hline 19 & Linear/splash & Linear & - & + \\
\hline 20 & Splash & Splash & - & + \\
\hline
\end{tabular}

SPECT-CT/lymphoscintigraphy results are described at baseline and 1 year after lymphovenous anastomosis (LVA). Results are marked as follows: -: No detectable migration of the tracer from the site of injection to the groin or axilla, lymphatic leakage or dermal backflow; +: The report of new images of lymphatic flow along the limb; ++: The presence of lymph nodes at any point in the limb; ${ }^{*}$ The presence of extraanatomical lymphatic flow; Note that fluorescence patterns are detected $15 \mathrm{~min}$ after injection and SPECT-CT/lymphoscintigraphy images are obtained $3 \mathrm{~h}$ after injection. This evaluation was performed the day before LVA and 1 year after it. Patterns are labeled according to Yamamoto's dermal backflow classification ${ }^{[11]}$

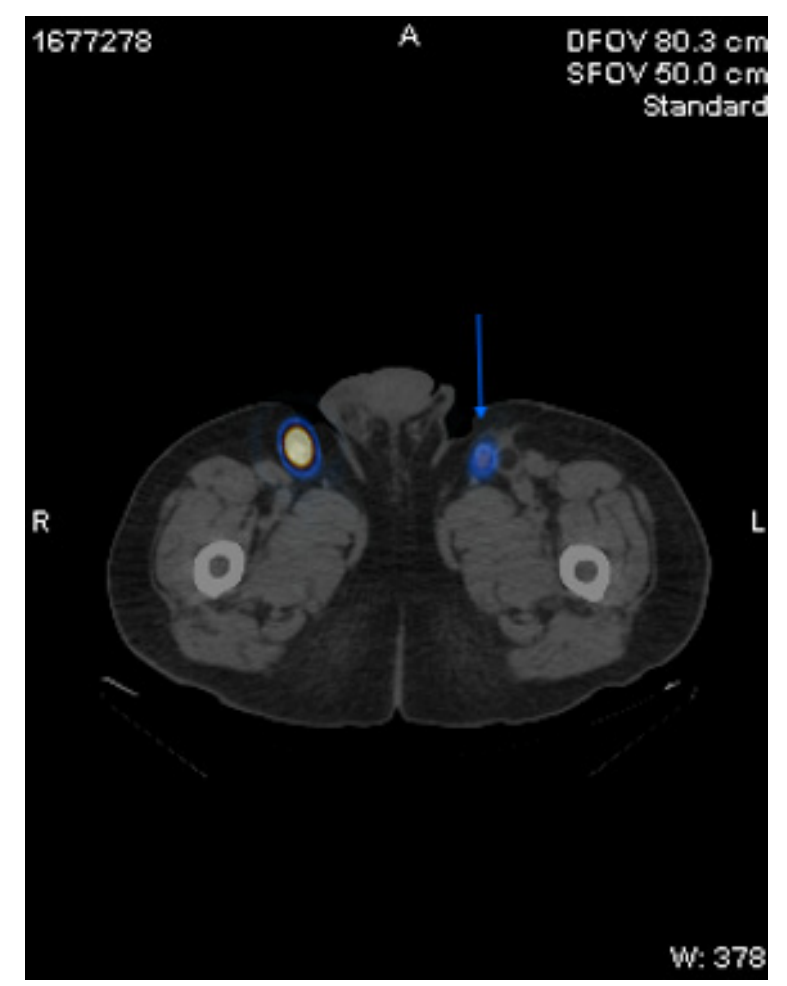

Figure 3. Pre-operative lympho-SPECT showing a lymph node at the root of the affected limb (left limb) that is smaller than the contralateral one (blue arrow). 


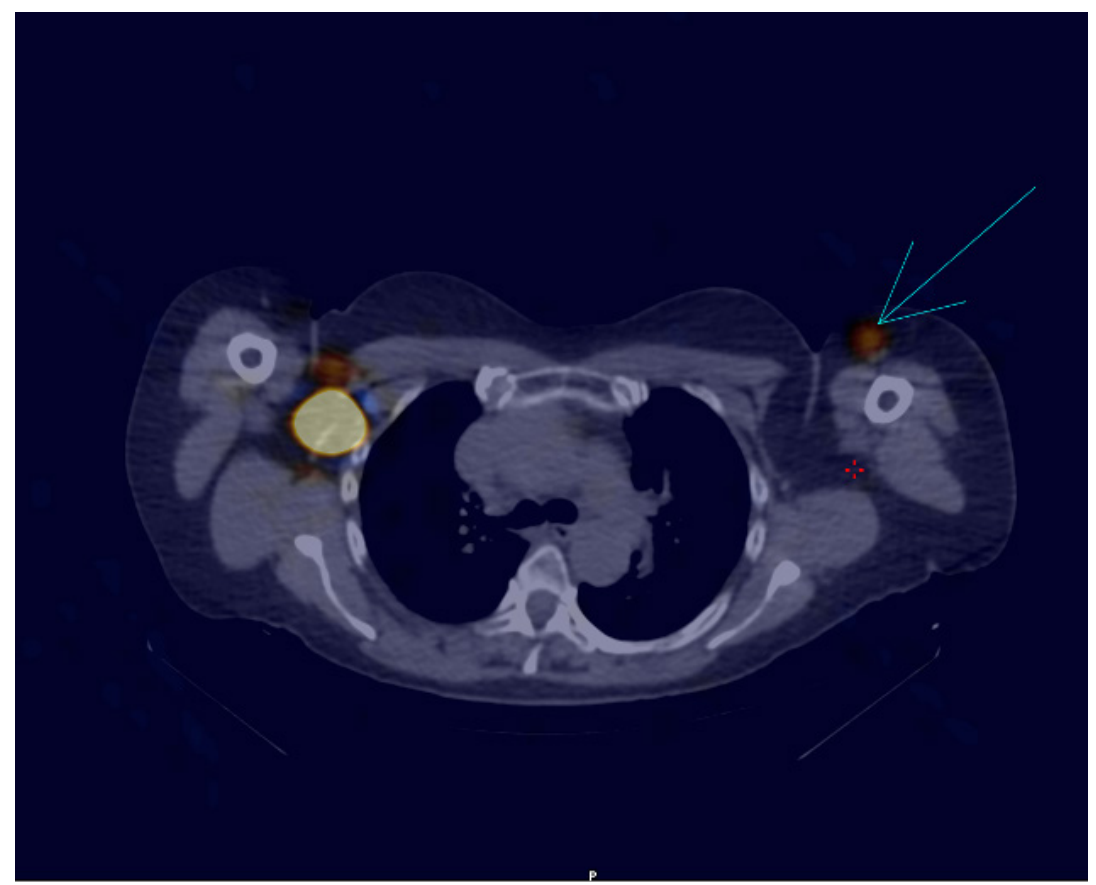

Figure 4. New lymph node at the arm of the patient with lymphedema of the left upper limb, at one year after lymphovenous anastomosis (blue arrow). The contralateral side (right axilla) is marking a functional nodal group

Figure 5 shows that in patient number 8, the stardust pattern had changed and new reticular and linear superficial patterns were observed after LVA. When lympho-SPECT/CT was correlated with these images, new images that were not visible before surgery could be observed.

The Spearman correlation coefficient was significantly negative when circumferences and lympho-SPECTCT were tested $(P=0.02)$.

\section{DISCUSSION}

Lymphoscintigraphy is a specific, simple and reliable technique that offers useful information of lymphatic function, allowing the examiner to detect lymphatic flow obstruction, dilated vessels, collateral lymphatic flow and the presence, malfunction or absence of lymph nodes ${ }^{[8-10]}$. Dermal uptake is a pathognomonic sign of lymphedema in limbs, and this finding is seen in most cases ${ }^{[11,12]}$. However, lymphoscintigraphy has limitations such as a two-dimensional view that does not allow projection onto anatomical landmarks. On the other hand, some artefacts can be observed when fibrosis, dermal backflow or accumulation in deeper vessels is seen ${ }^{[13]}$.

SPECT-CT is currently combined with lymphoscintigraphy for the detection of sentinel lymph nodes in the diagnosis of several tumours ${ }^{[14]}$. In lymphedema, SPECT-CT/lymphography systems provide integrated functional and morphological information, which allows better localization of the depth of vessels and lymph nodes. It is useful for accurate evaluation of anatomical differences between lymphatic vessels and veins and as well, it can provide better understanding of dermal backflow ${ }^{[15]}$.

SPECT-CT might be useful for predicting treatment efficacy when staging lymphedema ${ }^{[16-18]}$. In fact, combined CT-imaging has allowed better comprehension of the pathophysiology of lymphedema. Many studies have also proposed using SPECT-CT imaging beyond diagnosis by reporting its value in microsurgical treatment, which is essential for understanding the behavior of the lymphatic system after LVA or lymph node transfer. 

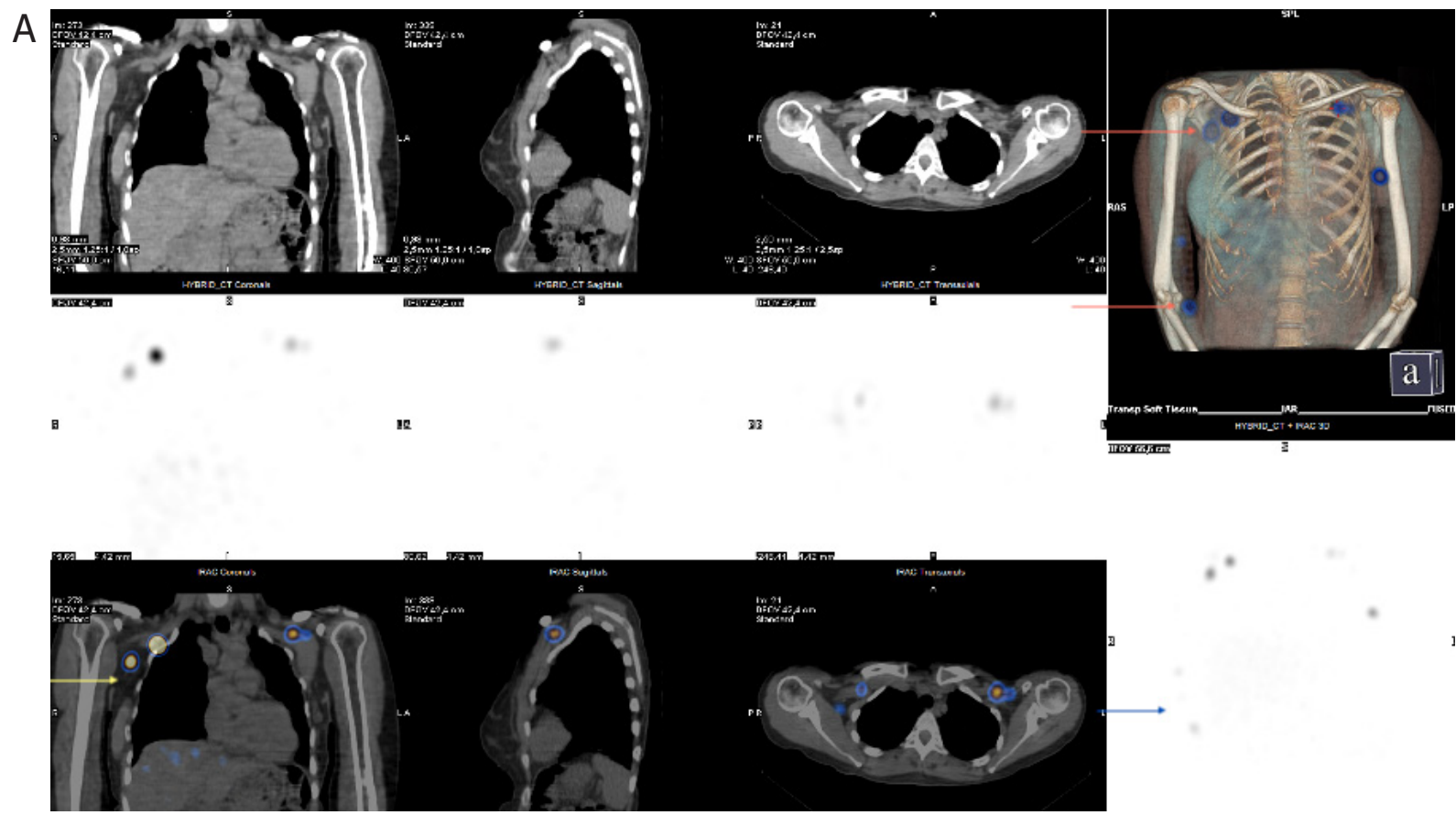

B video_01.mp4 - Reproductor multimedia VLC

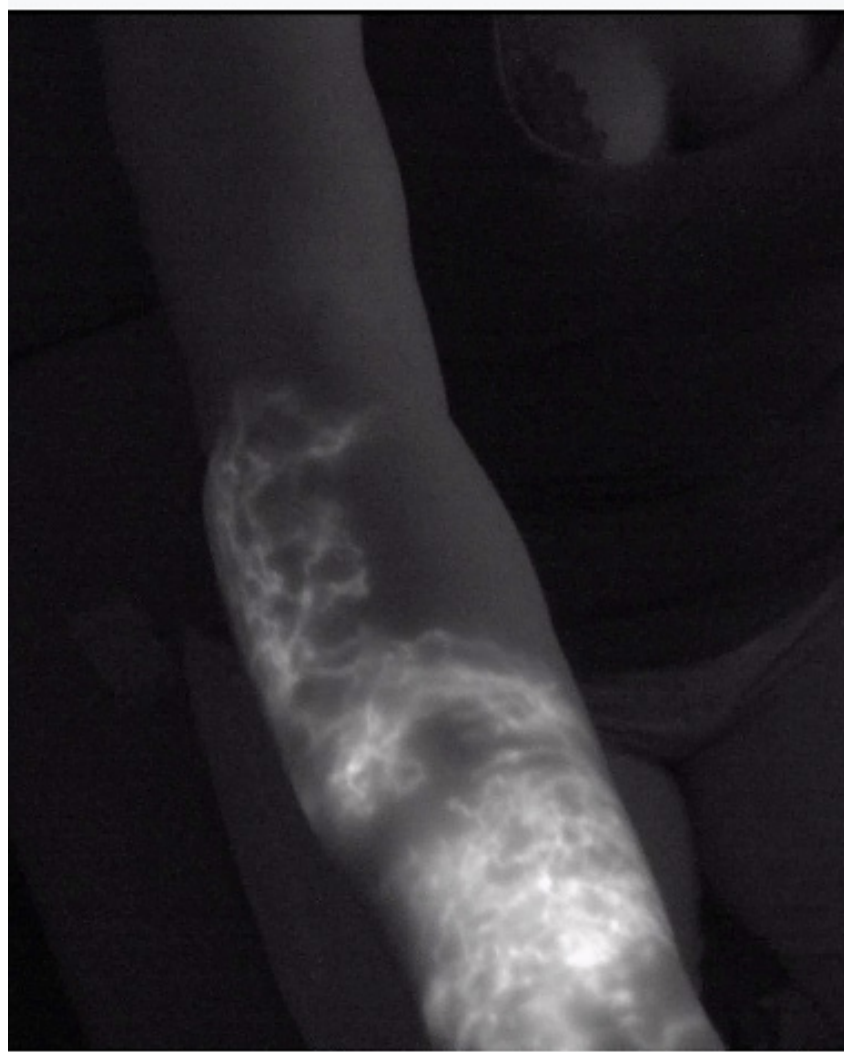

Figure 5. Lympho-SPECT, lymphography and ICG evaluation in patient 8. A: Software reconstruction showing a positive node in the right infraclavicular area and elbow after lymphovenous anastomosis (LVA). Orange and yellow arrows are marking the new activity. We can also observe the right breast implant for reconstruction, as well as lymphography under three-dimensional reconstruction (blue arrow); B: development of linear and reticular patterns one year after LVA in the forearm of this patient 
In this study, we evaluated the changes in lymphatic drainage one year after LVA and corroborated the effectiveness of surgical treatment. Our study population included a small group of randomly selected patients that were included in a prospective study. After analysis of lympho-SPECT-CT findings, we reported that in a majority of patients, regardless of the degree of lymphedema, there was evidence corresponding to new lymphatic circulation in images of the limbs (upper and lower limbs). We believe that LVA was not only useful in the reestablishment of lymphatic drainage through the venous by-passes, but also in developing collateral circulation (especially in two cases with extraanatomical landmarks). This could be explained in relation to the activation of new routes of drainage ${ }^{[19]}$. Cases with new lymph nodes may indicate that drainage is greater, as has been demonstrated in experimental models ${ }^{[20]}$. Interestingly, ICG findings are in concordance with these positive landmarks but we could not compare qualitative variables with quantitative ones to perform a better statistical analysis. In seven cases, we clearly observed that the linear pattern ${ }^{[2,22]}$ obtained after LVA was accompanied by more remarkable findings on lymphoSPECT-CT.

On the other hand, we were able to evaluate the correlation between clinical parameters such as the volume of the limbs and findings on lympho-SPECT. In this case, we observed an inverse correlation coefficient with statistical significance, indicating that the reduction of limb volume that is observed after LVA is associated with a significant increase in the number of landmarks that support improvement and reestablishment of lymphatic drainage. There are many factors influencing the final result. This is a heterogeneous group of patients, with different ages and time of evolution of lymphedema. Our work includes both upper and lower limb lymphedema of different ISL stages, which make comparisons difficult. It has also been demonstrated that LVA studies have a poor quality of evidence and high risk of bias, and therefore, it is difficult to draw reliable conclusions on the clinical effectiveness of $\mathrm{LVA}^{[23]}$.

Nevertheless, LVA seems to be safe for the treatment of primary and secondary lymphedema. If a reduction of volume can be demonstrated after LVA, we may think that lymphatic drainage has improved. In order to prove this, nuclear medicine testing would be an objective method to do so. If we are able to demonstrate such improvement by means of functional and anatomical landmarks (the development of new lymph nodes or the establishment of collateral circulation) that were not visible before surgery, we would then be in a position to confirm that surgery has been effective as it has been demonstrated that 99mTcphytate lymphoscintigraphy with SPECT-CT can provide both functional and morphological information simultaneously in patients with upper-limb lymphedema ${ }^{[24]}$. Using this modality, SPECT-CT may accurately reflect lymphodynamic conditions of the limbs after LVA. Furthermore, the surgical therapeutic efficacy could be estimated quantitatively by comparing pre- and postoperative findings.

In conclusion, our results have showed a postoperative decrease in volume of limbs with lymphedema that correlated inversely with lympho-SPECT/CT findings. Lympho-SPECT/CT provided additional information related to the accurate identification and anatomical location of lymphatic structures that were not observed before reconstructive surgery. It should be a complementary test to conventional lymphoscintigraphy.

\section{DECLARATIONS}

\section{Authors' contributions}

The author contributed solely to the article.

\section{Availability of data and materials}

Data supporting these findings can be found. Data can be deposited into the data repositories of the hospital, which can be accessed but not sent externally except for legal reasons. 


\section{Financial support and sponsorship}

None.

\section{Conflicts of interest}

The author declared that there are no conflicts of interest.

\section{Ethical approval and consent to participate}

The study was approved by the Ethics Committee and conforms to the Helsinki Declaration. Patients signed and gave informed consent for every operation and participation.

\section{Consent for publication}

The authors and patients gave consent for publication.

\section{Copyright}

(C) The Author(s) 2020 .

\section{REFERENCES}

1. Koshima I, Kawada S, Moriguchi T, Kajiwara Y. Ultrastructural observation of lymphatic vessels in lymphedema in human extremities. Plast Reconstr Surg 1996;97:397-405.

2. Koshima I, Inagawa K, Urushibara K, Moriguchi T. Supermicrosurgical lymphaticovenular anastomosis for the treatment of lymphedema in the upper extremities. J Reconstr Microsurg 2000;16:437-42.

3. Koshima I, Nanba Y, Tsutsui T, Takahashi Y, Itoh S. Long-term follow-up after lymphaticovenular anastomosis for lymphedema in the legs. J Reconstr Microsurg 2003;19:209-15.

4. Koshima I, Nanba Y, Tsutsui T, Takahashi Y, Itoh S, et al. Minimal invasive lymphaticovenular anastomosis under local anesthesia for leg lymphedema: is it effective for stage III and IV? Ann Plast Surg 2004;53:261-6.

5. Blum KS, Radtke C, Knapp WH, Pabst R, Gratz KF. SPECT-CT: a valuable method to document the regeneration of lymphatics and autotransplanted lymph node fragments. Eur J Nucl Med Mol Imaging 2007;34:1861-7.

6. Likert R. A technique for the measurement of attitudes. Arch Psychol 1932;22:55.

7. Yamamoto T, Narushima M, Doi K, Oshima A, Ogata F, et al. Characteristic indocyanine green lymphography findings in lower extremity lymphedema: the generation of a novel lymphedema severity staging system using dermal backflow patterns. Plast Reconstr Surg 2011;127:1979-86.

8. Szuba A, Shin WS, Strauss HW, Rockson S. The third circulation: radionuclide lymphoscintigraphy in the evaluation of lymphedema. J Nucl Med 2003;44:43-57.

9. Williams WH, Witte $\mathrm{Cl}$, Witte $\mathrm{MH}, \mathrm{McNeill}$ GC. Radionuclide lymphangioscintigraphy in the evaluation of peripheral lymphedema. Clin Nucl Med 2000;25:451-64.

10. Maegawa K, Miami T, Yamamoto Y, Satake T, Kobayashi S. Types of lymphoscintigraphy and indications for lymphaticovenous anastomosis. Microsurgery 2010;30:437-42.

11. Witte CL, Witte MH, Unger EC, Williams WH, Bernas MJ, et al. Advances in imaging of lymph flow disorders. Radiographics 2000;20:1697-19.

12. International Society of Lymphology. The diagnosis and treatment of peripheral lymphedema: 2013 consensus document of the international society of lymphology. Lymphology 2013;46:106-19.

13. Baulieu F, Bourgeois P, Maruani A, Belgrado JP, Tauveron V, et al. Contributions of SPECT/CT imaging to the lymphoscintigraphic investigations of the lower limb lymphedema. Lymphology 2013;46:106-19.

14. Cheville AL, Brinkmann DH, Ward SB, Durski J, Laack NN, et al. The addition of SPECT/CT lymphoscintigraphy to breast cancer radiation planning spares lymph nodes critical for arm drainage. Intl J Rad Onc Bio Phys 2013;85:971-7.

15. Iimura T, Fukushima Y, Kumita S, Ogawa R, Hyakusoku H. Estimating lymphodynamic conditions and lymphovenous anastomosis efficacy using 99mTc-phytate lymphoscintigraphy with SPECT-CT in patients with lower-limb lymphedema. Plast Reconstr Surg Glob Open 2015;3:e404.

16. Weiss M, Baumeister RG, Frick A, Wallmichrath J, Bartenstein P, et al. Primary lymphedema of the lower limb: the clinical utility of single photon emission computed tomography/CT. Korean J Radiol 2015;16:188-95.

17. Pecking AP, Albérini JL, Wartski M, Edeline V, Cluzan RV. Relationship between lymphoscintigraphy and clinical findings in lower limb lymphedema (LO): toward a comprehensive staging. Lymphology 2008;41:1-10.

18. Campisi C, Boccardo F. Microsurgical technique for lymphedema treatment: Derivative lymphatic-venous microsurgery. World J Surg 2004;28:609-13.

19. Brouwer OR, Vermeeren L, van der Ploeg IM, Valdés Olmos RA, Loo CE, et al. Lymphoscintigraphy and SPECT/CT in multicentric and multifocal breast cancer: does each tumour have a separate drainage pattern? Results of a Dutch multicentre study (MULTISENT). Eur J Nucl Med Mol Imaging 2012; 39:1137-43. 
20. Blum KS, Hadamitzky C, Gratz KF, Pabst R. Effects of autotransplanted lymph node fragments on the lymphatic system in the pig model. Breast Cancer Res Treat 2010;120:59-66.

21. Narushima M, Yamamoto T, Ogata F, Yoshimatsu H, Mihara M, et al. Indocyanine green lymphography findings in limb lymphedema. J Reconstr Microsurg 2016;32:72-9.

22. Chen WF, Zhao H, Yamamoto T, Hara H, Ding J. Indocyanine green lymphographic evidence of surgical efficacy following microsurgical and supermicrosurgical lymphedema reconstructions. J Reconstr Microsurg 2016;32:688-98.

23. Rosian K, Michal Stanak M. Efficacy and safety assessment of lymphovenous anastomosis in patients with primary and secondary lymphoedema: a systematic review of prospective evidence. Microsurgery 2019;39:763-72.

24. Visconti G, Hayashi A, Tartaglione G, Salgarello M, Yamamoto T. Innovative surgical treatment of peripheral lymphedema after breast cancer surgery. Transl Cancer Res 2018;7:S365-78. 